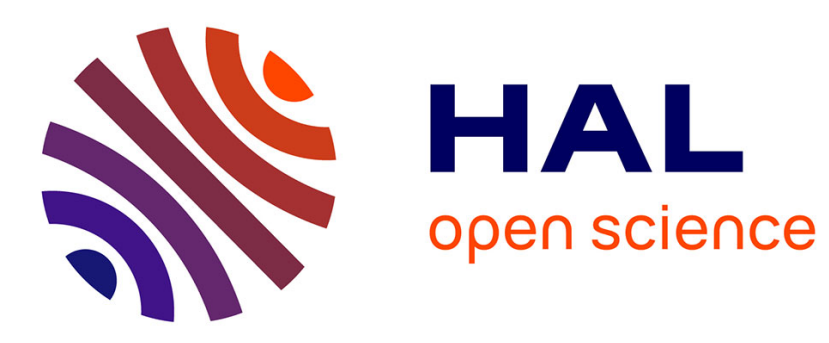

\title{
Identification of Simple Elements in Max-Algebra: Application to SISO Discrete Event Systems Modelisation
}

F. Gallot, Jean-Louis Boimond, Laurent Hardouin

\section{- To cite this version:}

F. Gallot, Jean-Louis Boimond, Laurent Hardouin. Identification of Simple Elements in Max-Algebra: Application to SISO Discrete Event Systems Modelisation. ECC'97, Jul 1997, Bruxelles, Belgium. pp.x-x. hal-00843987

\section{HAL Id: hal-00843987 \\ https://hal.science/hal-00843987}

Submitted on 12 Jul 2013

HAL is a multi-disciplinary open access archive for the deposit and dissemination of scientific research documents, whether they are published or not. The documents may come from teaching and research institutions in France or abroad, or from public or private research centers.
L'archive ouverte pluridisciplinaire HAL, est destinée au dépôt et à la diffusion de documents scientifiques de niveau recherche, publiés ou non, émanant des établissements d'enseignement et de recherche français ou étrangers, des laboratoires publics ou privés. 


\title{
IDENTIFICATION OF SIMPLE ELEMENTS IN MAX- ALGEBRA: APPLICATION TO SISO DISCRETE EVENT SYSTEMS MODELISATION
}

\author{
F. GALLOT, J.L. BOIMOND, L. HARDOUIN
}

\author{
L.I.S.A., 62, Avenue Notre Dame du Lac, 49000 ANGERS, FRANCE \\ Fax number: 0241365735 - E-mail: [frederic.gallot, jean-louis.boimond, laurent.hardouin]@istia.univ-angers.fr
}

Keywords: Discrete-event systems, Identification, Modelling, Max-algebra.

\begin{abstract}
We propose to modelise the time behaviour of SISO discreteevent systems linear in Max-algebra. The method is inspired from the conventional linear system theory: from the impulse response, the model is obtained by using a decomposition of the system into a sum of first order sub-systems. From an ARMA form, we compute the parameters of the model by using both results of residuation theory to minimise an error criterion and decomposition of periodic series.
\end{abstract}

\section{Introduction}

In performance evaluation systems [1], [5] or in control system setting [2], [4], we need a mathematical model to characterise systems properties.

We are interested in modelling SISO discrete-event systems (DES) whose behaviours can be described by timed-event graphs (TEG) [1] (a sub-class of Petri nets which can represent synchronisation constraints). It is well known that a TEG can be linearly described in Max-algebra which enables finding analogies with the conventional linear systems theory.

Most works on modelling deal with the minimality of the model [7], [10], [12]. The present work does not care about this problem. We are only interested in developing a simple method to compute the parameters of the transfer relation by the analyse of the DES impulse response. Such an approach has been already proposed in [3]. In this paper, we propose a more convenient modelisation method, in the sense that it does not need to have an estimation of the beginning periodic behaviour of the impulse response. The approach is based on the decomposition property of a linear system into a sum of finite simple elements (under the form $d \gamma^{c}\left(\tau \gamma^{v}\right)^{*}$ ) which is reminiscent of the decomposition of a linear system into a sum of first order simple elements in conventional linear system theory. Then the proposed modelling method individually identifies these simple elements in order to have a model (expressed as a periodic form).
The paper is organised as follows: the second part recalls the few linear algebraic results needed here. The third part presents the identification method in Max-algebra which is both based on the Residuation theory [1, sec. 4.4], [6] and on the decomposition of periodic series [8], [11]. A practical modelling procedure is presented in part 4 and a short example is given in part 5 .

\section{Linear algebraic representation}

Some results related to dioids, residuation, TEG and rational series are briefly presented in this section. For a general survey see [1], [5], [8].

\subsection{Dioids}

Definition 1 (dioid): A dioid $D$ is a set endowed with two internal operations denoted $\oplus$ (addition) and $\otimes$ (multiplication), both associative and both having neutral elements denoted $\varepsilon$ and $e$ respectively, such that: $\oplus$ is commutative and idempotent $(\forall a, a \oplus a=a), \otimes$ is distributive with respect to $\oplus$, and $\varepsilon$ is absorbing for $\otimes$ $(\forall a, \varepsilon \otimes a=a \otimes \varepsilon=\varepsilon)$.

Note: The symbol $\otimes$ will be omitted subsequently.

Definition 2 (commutative dioid): A dioid is commutative iff the product $\otimes$ is commutative.

Definition 3 (natural order): In a dioid, the following relation denoted $\succeq$ is a (partial) order relation:

$$
a \succeq b \Leftrightarrow a=a \oplus b
$$

Definition 4 (complete diod): A dioid is complete iff it is closed for infinite sums and if the product $\otimes$ distributes over infinite sums. There is a maximal element of the dioid denoted by $T$ (the sum of all elements) which is absorbing for addition $(\forall a, a \oplus T=T \oplus a=T)$.

Example: The set $\mathrm{Z} \cup\{-\infty,+\infty\}$ with the operations $\oplus$ and $\otimes$ defined by $a \oplus b=\max \{a, b\}, a \otimes b=a+b$ is a complete commutative dioid denoted $\bar{Z}_{\max }$ with $\varepsilon=-\infty, T=+\infty$ and $e=0$. 
Definition 5 (lower bound): In a complete dioid $D$, since there is a bottom element $\varepsilon$, the lower bound can be contructed for any subset $C$ of $D$. If $C=\{a, b\}$ then its lower bound is denoted $a \wedge b$. One has the following equivalence:

$$
a \succeq b \Leftrightarrow a=a \oplus b \Leftrightarrow b=a \wedge b
$$

Definition 6 (isotone mapping): A mapping $f: D \rightarrow C$, where $D$ and $C$ are ordered sets, is isotone if:

$$
\forall a, b \in D, a \succeq b \Rightarrow f(a) \succeq f(b)
$$

\subsection{Residuation}

Theorem 1 ([1, sec. 4.4.2]): Let $f$ be an isotone mapping from the complete dioid $D$ into the complete dioid $C$. The following three statments are equivalent:

- For all $b \in C$, there exists a greatest subsolution to the equation $f(x)=b$;

- $f(\varepsilon)=\varepsilon$ and $f$ is lower semi-continuous, i.e.,

$$
f\left(\bigoplus_{x \in X \subset D} x\right)=\bigoplus_{x \in X \subset D} f(x) \text {; }
$$

- There exists an isotone mapping $f^{\#}$ from $C$ into $D$ such that:

$$
\begin{aligned}
& f \circ f^{\#} \preceq I_{C} \quad \text { (identity of } C \text { ); } \\
& f^{\#} \circ f \succeq I_{D} \quad \text { (identity of } D \text { ). }
\end{aligned}
$$

Consequently, $f^{\#}$ is unique. When $f$ satisfies these properties, it is said to be residuated and $f^{\#}$ is called its residual.

This theorem can be applied to the mapping $x \mapsto a \otimes x$ in a complete dioid. The residual mapping will be denoted $y \mapsto a \backslash y$.

\subsection{Matrix residuation}

Starting from a "scalar" dioid $D$, consider square $n \times n$ matrices with entries in $D$. the sum and product of matrices are defined conventionally after the sum and product of scalars in $D$. The set of $n \times n$ matrices supplied with these two operations is also a dioid denoted by $D^{n \times n}$.

Theorem 2: Let $A \in D^{m \times n}$ and $B \in D^{m \times p}$, the residual of $B$ by $A$ will be denoted $C=A \mid B, C \in D^{n \times p}$ :

$$
C_{i j}=\bigwedge_{k=1}^{m}\left(A_{k i} \backslash B_{k j}\right)
$$

\subsection{The equation $x=a x \oplus b$ and the star operation}

Consider the equation:

$$
x=a x \oplus b
$$

and let $a^{*}=e \oplus a \oplus a^{2} \oplus \ldots$

Theorem 3 ([1, sec. 4.5.3]): Consider Eq. (1) with $a$ and $b$ given in a complete dioid $D$. Then,
- $a^{*} b$ is the least solution of (1);

- every solution $x$ of (1) satisfies $x=a^{*} x$.

\subsection{TEG and daters approach}

Event graphs are a particular class of Petri nets in which each place has exactly one upstream and one downstream transition. In TEG, the holding time of a token in a place is the time a token must spend in the place before contributing to the enabling of the downstream transitions.

For a transition labelled $x$, we define the non decreasing mapping called dater $k \mapsto x(k)$ where $x(k)$ is the date when the $k^{\text {th }}$ firing of the transition $x$ has occured.

Let us consider the manufacturing system described by the TEG of Fig. 1.

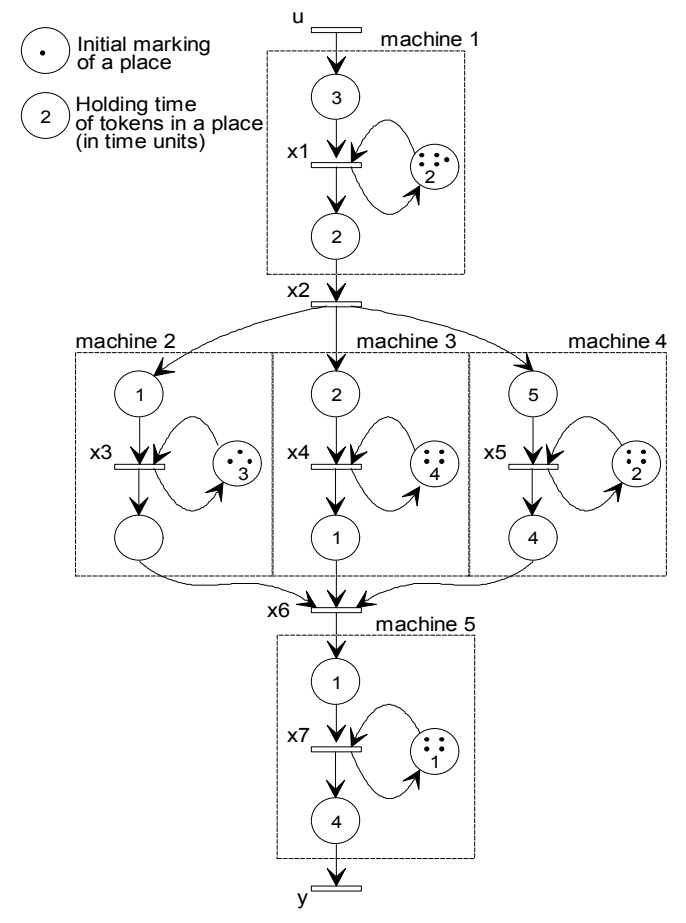

Fig 1: A TEG.

For example, (dater) equations obtained for transitions $x_{1}$ and $x_{6}$ in $\bar{Z}_{\max }$ are:

$x_{1}(k)=3 u(k) \oplus 2 x_{1}(k-5)$

$x_{6}(k)=x_{3}(k) \oplus 1 x_{4}(k) \oplus 4 x_{5}(k)$

Using the analogue of the z-transform of conventional linear system theory, we introduce the $\gamma$-transform of daters $(\gamma$ can be interpreted as backward shift operator: $\gamma v(k)=v(k-1))$. The $\gamma$-transform of a dater $v$ is defined as:

$$
V(\gamma)=\bigoplus_{k \in Z} \gamma^{k} v(k)
$$


For example, the Eqs. (2) can be transposed in the dioid of non decreasing formal series $\bar{Z}_{\max }[[\gamma]]$ (set $\mathrm{Z} \cup\{-\infty,+\infty\}$ with the laws $\oplus$ and $\otimes$ defined respectively by max and + of formal series in $\gamma$ :

$X_{1}(\gamma)=3 U(\gamma) \oplus 2 \gamma^{5} X_{1}(\gamma)$

$X_{6}(\gamma)=X_{3}(\gamma) \oplus 1 X_{4}(\gamma) \oplus 4 X_{5}(\gamma)$

Usually, TEG are described by the following linear states equations:

$$
\left\{\begin{array}{l}
\underline{X}(\gamma)=A \underline{X}(\gamma) \oplus B \underline{U}(\gamma) \\
\underline{Y}(\gamma)=C \underline{X}(\gamma)
\end{array}\right.
$$

For the TEG given in Fig. 1 we have:

$$
\begin{aligned}
& \underline{X}(\gamma)=\left[\begin{array}{ccccccc}
2 \gamma^{5} & \varepsilon & \varepsilon & \varepsilon & \varepsilon & \varepsilon & \varepsilon \\
2 & \varepsilon & \varepsilon & \varepsilon & \varepsilon & \varepsilon & \varepsilon \\
\varepsilon & 1 & 3 \gamma^{3} & \varepsilon & \varepsilon & \varepsilon & \varepsilon \\
\varepsilon & 2 & \varepsilon & 4 \gamma^{4} & \varepsilon & \varepsilon & \varepsilon \\
\varepsilon & 5 & \varepsilon & \varepsilon & 2 \gamma^{4} & \varepsilon & \varepsilon \\
\varepsilon & \varepsilon & e & 1 & 4 & \varepsilon & \varepsilon \\
\varepsilon & \varepsilon & \varepsilon & \varepsilon & \varepsilon & 1 & \gamma^{4}
\end{array}\right] X(\gamma) \oplus\left[\begin{array}{l}
3 \\
\varepsilon \\
\varepsilon \\
\varepsilon \\
\varepsilon \\
\varepsilon \\
\varepsilon
\end{array}\right] U(\gamma) \\
& Y(\gamma)=\left[\begin{array}{lllllll}
\varepsilon & \varepsilon & \varepsilon & \varepsilon & \varepsilon & \varepsilon & 4
\end{array}\right] \underline{X}(\gamma)
\end{aligned}
$$

According to theorem 3 we conclude that:

$$
\underline{X}(\gamma)=A^{*} B U(\gamma) \text { and then } Y(\gamma)=C A^{*} B U(\gamma)
$$

which leads to the transfer relation:

$$
Y(\gamma)=h(\gamma) U(\gamma) \text { with } h(\gamma)=C A^{*} B
$$

If input $U(\gamma)$ of the system is an impulsion (transition $u$ is infinitely fired at time 0$)$ then output $Y(\gamma)$ can be considered as impulse response $h(\gamma)$ of the system.

For example transfer relation $h(\gamma)$ corresponding to Eqs. 4 leads to the impulse response illustrated in Fig. 2.

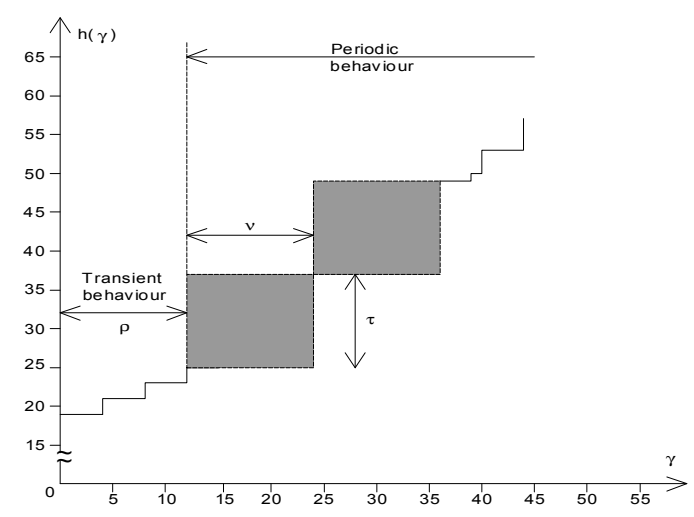

Fig. 2: Impulse response of the transfert relation $h(\gamma)$.

\subsection{Rational series over $\bar{Z}_{\max }[[\gamma]]$}

Definition 7 (rationality): A series $s(\gamma) \in \bar{Z}_{\max }[[\gamma]]$ is rational if its expression is composed of a finite number of operation $\otimes, \oplus$ and $*$.

Definition 8 (periodicity): A series $s(\gamma) \in \bar{Z}_{\max }[[\gamma]]$ is periodic if there exists two polynomials $p(\gamma), q(\gamma)$ and a monomial $\tau \gamma^{v} \in \bar{Z}_{\max }[[\gamma]]$ such that:

$s(\gamma)=p(\gamma) \oplus q(\gamma)\left(\tau \gamma^{\nu}\right)^{*}$
with $p(\gamma)=\bigoplus_{i=0}^{\rho-1} p_{i} \gamma^{i}, q(\gamma)=\bigoplus_{j=0}^{\mathrm{v}-1} q_{j} \gamma^{j+\rho}$

Definition 9 (realisabilty): A series $s(\gamma) \in \bar{Z}_{\max }[[\gamma]]$ is realisable if it can be written as: $s(\gamma)=C A^{*} B$.

Theorem 4 [5]: For $s(\gamma) \in \bar{Z}_{\max }[[\gamma]]$, the following three statements are equivalent:

- $s(\gamma)$ is rational;

- $s(\gamma)$ is periodic;

- $s(\gamma)$ is realisable.

For example the transfer relation $h(\gamma)$ corresponding to Eqs. 4 can be written under the following periodic expression:

$$
\begin{aligned}
h(\gamma)= & 19 \oplus 21 \gamma^{4} \oplus 23 \gamma^{8} \\
& \oplus\left(25 \gamma^{12} \oplus 26 \gamma^{15} \oplus 29 \gamma^{16} \oplus 33 \gamma^{20}\right)\left(12 \gamma^{12}\right)^{*}
\end{aligned}
$$

The periodic expression:

$$
h(\gamma)=p(\gamma) \oplus q(\gamma)\left(\tau \gamma^{v}\right)^{*}
$$

traduces that the pattern represented by $q(\gamma)$ is indefinitely reproduced because the multiplication by $\tau \gamma^{\nu}$ symbolises a $v$ unit abscissa shift and a $\tau$ unit ordinate shift. This periodic behaviour begins after a transient behaviour which may be represented by $p(\gamma)$.

Definition 10 (simple element): A series $s(\gamma) \in \bar{Z}_{\max }[[\gamma]]$ is a simple element if it can be written as: $s(\gamma)=d \gamma^{c}\left(\tau \gamma^{v}\right)^{*}$

Theorem 5 [5]: A series $s(\gamma) \in \bar{Z}_{\max }[[\gamma]]$ is rational iff it can be written as a finite sum of simple elements.

From theorems 4 and 5, we obviously see that a periodic series $s(\gamma) \in \bar{Z}_{\max }[[\gamma]]$ can be written as a finite sum of simple elements:

$$
p(\gamma) \oplus q(\gamma)\left(\tau \gamma^{v}\right)^{*}=\bigoplus_{i=1 \text { to } l} d_{i} \gamma^{c_{i}}\left(\tau_{i} \gamma^{v_{i}}\right)^{*}
$$


Remark 1 (minimal representation): Given an impulse response $h(\gamma)$ and $S=\left\{s_{i}(\gamma), \ldots, s_{l}(\gamma)\right\} \quad$ with $s_{i}(\gamma)=d_{i} \gamma^{c_{i}}\left(\tau_{i} \gamma^{v_{i}}\right)^{*}$, the minimal representation problem consists in finding a subset of $S$ with minimal cardinality $l_{\min }$ such that:

$$
h(\gamma)=\bigoplus_{i=1}^{l_{\min }} d_{i} \gamma^{c_{i}}\left(\tau_{i} \gamma^{v_{i}}\right)^{*} \quad(\text { see }[1, \text { sec. } 6.5 .4])
$$

Remark 2 [8]: A periodic series has one simplest periodic representation. This author gives some efficient algorithms to establish the simplest polynomials $p(\gamma)$ and $q(\gamma)$.

\section{Identification}

We plan to identify an ARMA model in max-algebra in order to compute in section 4 the parameters of the transfer relation $h(\gamma)$ (see Eq. 6).

Let us recall that in conventional discrete time system theory, the ARMA equation can be expressed as:

$$
y(k)=\sum_{j=1}^{n} a_{j} y(k-j)+\sum_{i=0}^{m} b_{i} u(k-i)
$$

To estimate the parameters of this equation, the following prediction error is classically defined:

$$
\varepsilon(k)=y_{G}(k)-\sum_{j=1}^{n} \hat{a}_{j} y_{G}(k-j)-\sum_{i=0}^{m} \hat{b}_{j} u(k-i)
$$

where $y_{G}$ is the measured output of the system and $\hat{a}_{1}, \ldots, \hat{a}_{n}, \hat{b}_{0}, \ldots, \hat{b}_{m}$ are the estimated parameters.

A common criterion, which is minimised for the set of parameters searched, is:

$$
J=\sum_{k} \varepsilon^{2}(k)
$$

This criterion is well known in least square method [9].

\subsection{Identification of max-algebra ARMA model}

By analogy with the previous method, let us consider the identification of the following ARMA equation in $\bar{Z}_{\max }[[\gamma]]$ :

$$
Y(\gamma)=\left[a_{1} \gamma \oplus \ldots \oplus a_{n} \gamma^{n}\right] Y(\gamma) \oplus\left[b_{0} \oplus \ldots \oplus b_{m} \gamma^{m}\right] U(\gamma)
$$

which corresponds in $\bar{Z}_{\max }$ to:

$$
y(k)=a_{1} y(k-1) \oplus . . . \oplus a_{n} y(k-n) \oplus b_{0} u(k) \oplus \ldots \oplus b_{m} u(k-m)
$$

or equivalently $y(k)=\underline{\varphi}_{k}^{T} \underline{\theta}$

with $\quad \underline{\varphi}_{k}^{T}=[y(k-1) \ldots y(k-n) \quad u(k) \ldots u(k-m)] \quad$ the regressive vector at event $k$ and $\underline{\theta}=\left[a_{1} \ldots a_{n} b_{0} \ldots b_{m}\right]^{T}$ the model parameters vector.
For event $k=i$ to $N \quad(i \geq n$ and $N$ is the number of data), we consider the matrix equation $\underline{Y}=M \underline{\theta}$ with $\underline{Y}=[y(i) \ldots y(N)]^{T}$ the model output vector and the matrix $M=\left[\underline{\varphi}_{i} \cdots \underline{\varphi}_{N}\right]^{T}$ of regressive vectors.

By analogy with the conventional linear system theory, the identification method considers the following prediction error:

$$
\varepsilon(k)=y_{G}(k)-\left(\underline{\varphi}_{k}^{T} \otimes \underline{\hat{\theta}}\right)
$$

with $\underline{\hat{\theta}}=\left[\hat{a}_{1} \ldots \hat{a}_{n} \hat{b}_{0} \ldots \hat{b}_{m}\right]^{T}$ the estimated parameters vector and $\quad \underline{\varphi}_{k}^{T}=\left[y_{G}(k-1) \ldots y_{G}(k-n) \quad u(k) \ldots u(k-m)\right] \quad$ the measured regressive vector at event $k$ with $y_{G}$ the measured system output.

By using the data of $u(k)$ and $y_{G}(k)$, with $k=i-n$ to $N$ $(i \geq n)$, we obtain the matrix expression:

$$
\underline{\varepsilon}=\underline{Y}_{G}-\left(M_{G} \otimes \underline{\hat{\theta}}\right)
$$

where $\underline{\varepsilon}=[\varepsilon(i) \ldots \varepsilon(N)]^{T}$ is the prediction error vector, $\underline{Y}_{G}=\left[y_{G}(i) \ldots y_{G}(N)\right]^{T}$ is the measured system output vector and $M_{G}$ is the matrix $\left[\underline{\varphi}_{i} \ldots \underline{\varphi}_{N}\right]^{T}$ of the measured regressive vectors.

Let us define a criterion $J$ as:

$$
J(\underline{\hat{\theta}})=\bigoplus_{k=i}^{N} \varepsilon(k) \quad \text { with } \varepsilon(k) \geq 0
$$

To determinate the estimated parameters vector $\hat{\hat{\theta}}$ which minimises this criterion, we consider a basic result of the Residuation theory [6] which states that $\underline{\hat{\theta}}=M_{G} \backslash \underline{Y}_{G}$ minimises $J(\underline{\hat{\theta}})$ and is the greatest solution of $\underline{Y}_{G} \succeq M_{G} \otimes \underline{\hat{\theta}}$.

\subsection{Identification of simple elements}

We study now the identification of simple elements.

Let us consider $Y(\gamma)=d \gamma^{c}\left(\tau \gamma^{v}\right)^{*} U(\gamma)$ (see def. 10) which leads by using theorem 3 to identify the following particular ARMA equation:

$$
Y(\gamma)=\tau \gamma^{v} Y(\gamma) \oplus d \gamma^{c} U(\gamma)
$$

which corresponds in $\bar{Z}_{\max }$ to

$$
y(k)=\tau y(k-v) \oplus d u(k-c)
$$

or equivalently $y(k)=\underline{\varphi}_{k}^{T} \underline{\theta}$ 
with $\underline{\varphi}_{k}^{T}=[y(k-v) \quad u(k-c)]$ and $\underline{\theta}=\left[\begin{array}{ll}\tau & d\end{array}\right]^{T}$.

In this paper, we consider an impulsion input $u$ :

$$
u(k)=\left\{\begin{array}{c}
e \text { if } k \geq 0 \\
\varepsilon \text { otherwise }
\end{array}\right.
$$

which corresponds to the input transition infinitely fired at the initial time.

According to the model structure and the impulsion input $U(\gamma)=e$, we have:

$$
Y(\gamma)=\tau \gamma^{v} Y(\gamma) \oplus d \gamma^{c}
$$

which means in $\bar{Z}_{\max }$ that $y(k)=\varepsilon$ for $k<c$ and

$$
y(c+i v)=y(c+i v+k) \quad i \geq 0,0 \leq k \leq v-1
$$

Hence, we compute the estimated vector $\underline{\hat{\theta}}=\left[\begin{array}{ll}\hat{\tau} & \hat{d}\end{array}\right]^{T} \quad\left(\begin{array}{l}c \text { and } \\ \text { a }\end{array}\right.$ $\mathrm{v}$ are fixed) by considering the measured output vector:

$$
\underline{Y}_{G}=\left[y_{G}(c) y_{G}(c+v) \ldots y_{G}(c+j v)\right]^{T}
$$

and the simplified matrix $M_{G}=\left[\begin{array}{lll}\underline{\varphi}_{c} & \cdots \underline{\varphi}_{c+j v}\end{array}\right]^{T}$ with $j=\operatorname{Sup}\{n \mid c+n v \leq N\}$ and $\underline{\varphi}_{c+s v}=\left[\begin{array}{ll}y_{G}(c+s v-v) & u(s v)\end{array}\right]^{T}$ where $y_{G}(k)=\varepsilon$ if $k<c$.

Then we can compute $\underline{\hat{\theta}}=M_{G} \backslash \underline{Y}_{G}$ which minimises the criterion $J=\bigoplus_{k=c}^{N} \varepsilon(k)$ with $\varepsilon(k) \geq 0$.

Remark 3: Since $\underline{\hat{\theta}}$ is computed in $\bar{Z}_{\max }$, $\hat{\hat{\theta}}_{i}=\bigwedge_{k}\left(M_{G_{k i}} \backslash \underline{Y}_{G_{k}}\right)=\bigwedge_{k}\left(\underline{Y}_{G_{k}}-M_{G_{k i}}\right), . i=\{1,2\}$.

\section{Practical modelling of $\boldsymbol{h}(\gamma)$}

The desired expression of the transfer relation $h(\gamma)$ is periodic which cannot be expressed as the ARMA form defined in the section 3.1.

A first solution to obtain $h(\gamma)$ has been proposed in [3] by using not directly this ARMA form. It consists in identifying the transient and the periodic parts of $h(\gamma)$ separately, which means to have an estimation of the transient part length. In this paper, this constraint is not necessary.

Let us consider the transfer relation $h(\gamma)$ as a finite sum (max) of simple elements $s_{i}(\gamma)$ (see Eq. 7):

$$
h(\gamma)=\bigoplus_{i=1}^{l} s_{i}(\gamma) \text { with } s_{i}(\gamma)=d_{i} \gamma^{c_{i}}\left(\tau_{i} \gamma^{v_{i}}\right)^{*}
$$

In other words we have:

$$
Y(\gamma)=\bigoplus_{i=1}^{l} Y_{i}(\gamma)=\bigoplus_{i=1}^{l} s_{i}(\gamma) U(\gamma)
$$

Under the assumption that the input is an impulsion $(U(\gamma)=e)$, it is possible to individually identify these simple elements (see section 3.2).

Practically, it consists in computing $v, \hat{\tau}, \hat{d}$ which minimises $J$ for $c$ fixed. This procedure is repeated by increasing $c$ until the sum of simple element outputs behaves as the system ouput (see the following algorithm).

$$
\begin{aligned}
& \underline{Y}_{M}=\underline{\varepsilon} \\
& c=0
\end{aligned}
$$

DO

$\underline{Y}_{M}$ prev $=\underline{Y}_{M}$

FOR $v=1$ to $(\mathrm{N}-\mathrm{c}) / 2$

$$
\text { Compute } \underline{\hat{\theta}}=M_{G} \backslash \underline{Y}_{G}=\left[\begin{array}{ll}
\hat{\tau} & \hat{d}
\end{array}\right]^{T}
$$

END_FOR

$\underline{Y}_{M}=\underline{Y}_{M} \operatorname{prev} \oplus \hat{d} \gamma^{c}\left(\hat{\tau} \gamma^{v}\right)^{*}$

IF $\underline{Y}_{M} \succeq \underline{Y}_{M}$ prev THEN the simple element is kept

$$
c=c+1
$$

UNTIL $\underline{Y}_{M}$ near $\underline{Y}_{G}$

Then we sum these simple elements thanks to the algorithm proposed in [8] in order to obtain the periodic series which characterises $h(\gamma)$.

\section{Example}

Let us apply results of section 4 to modelise the transfer relation $h(\gamma)$ corresponding to Fig. 2. In this way, we suppose to have the $N$ first $(N=44)$ measured data $Y_{G}$ represented in dotted line in Fig. 3. To illustrate the modelling procedure, we have also represented the simple element given by the algorithm for $c=12$.

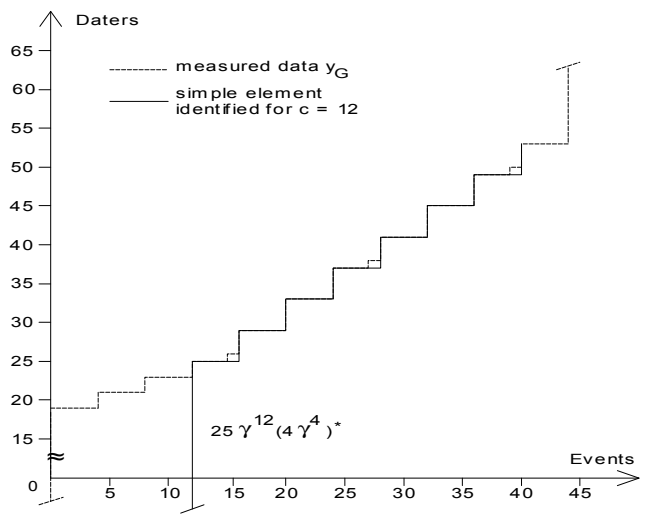

Fig 3 : Modelling procedure. 
The result of this modelling procedure yields to:

$$
\begin{gathered}
h(\gamma)=19 \gamma^{0}\left(18 \gamma^{24}\right)^{*} \oplus 21 \gamma^{4}\left(16 \gamma^{22}\right)^{*} \oplus 21 \gamma^{5}\left(12 \gamma^{15}\right)^{*} \\
\oplus 21 \gamma^{6}\left(12 \gamma^{14}\right)^{*} \oplus 21 \gamma^{7}\left(8 \gamma^{9}\right)^{*} \oplus 23 \gamma^{8}\left(18 \gamma^{20}\right)^{*} \\
\oplus 23 \gamma^{11}\left(15 \gamma^{16}\right)^{*} \oplus 25 \gamma^{12}\left(4 \gamma^{4}\right)^{*} \oplus 26 \gamma^{15}\left(12 \gamma^{12}\right)^{*}
\end{gathered}
$$

Using algorithm proposed by [8] the transfer relation can be written under the simplest representation of the periodic series defined in section 2:

$$
\begin{aligned}
h(\gamma) & =19 \oplus 21 \gamma^{4} \oplus 23 \gamma^{8} \\
& \oplus\left(25 \gamma^{12} \oplus 26 \gamma^{15} \oplus 29 \gamma^{16} \oplus 33 \gamma^{20}\right)\left(12 \gamma^{12}\right)^{*}
\end{aligned}
$$

which corresponds to the transfer relation of the system.

Remark 4: The given algorithm detects the periodic part of the impulse response when it appears twice in the measured data. If not, it will return many degenerated simple elements of the form $d \gamma^{c}\left(0 \gamma^{v}\right)^{*}$.

\section{Conclusion}

We have proposed a practical modelling method of linear SISO DES in Max-Algebra. This method offers analogies with conventional linear system theory: the impulse response is considered as a sum of simple elements which are individually identified. We have improved the method given in [3] in the sense that it does not need to have an estimation of the transient part of the transfer relation $h(\gamma)$.

Let us note that the algorithm does not guarantee the minimal representation of the system (see remark 1), nevertheless we obtain the simplest representation of the periodic series which characterises the transfer relation $h(\gamma)$ (see remark 2).

\section{References}

[1] Baccelli F., Cohen G., Olsder G.J., Quadrat J.P., "Synchronization and Linearity. An Algebra for Discrete Event Systems", New York: Willey, (1992).

[2] Boimond J.L., "Internal Model Control of Discrete Event Processes in the Max-Algebra", ECC 93, pp. 150-157, Groningen, (1989).

[3] Boimond J.L., Hardouin L., Chiron P., "A Modeling Method of SISO Discrete Event Systems in MaxAlgebra", ECC 95, pp. 2023-2026, Roma, (1995).

[4] Boimond J.L., Ferrier J.L., "Internal Model Control and Max-Algebra: Controller Design", IEEE TAC, 41, n³, pp. 457-461, (march 1996).
[5] Cohen G., Dubois D., Quadrat J.P., Viot M., "Algebraic Tools for the Performance Evaluation of Discrete Event Systems", IEEE TAC, 77, n¹, pp.39-58, (january 1989).

[6] Cuninghame-Green R., "Minimax Algebra", Lecture Notes in Economics an Mathematical Systems, SpringerVerlag, $\mathbf{n}^{\circ} \mathbf{1 6 6}$, (1979).

[7] De Schutter B., "Max Algebraic System Theory for Discrete Event Systems", Thesis, Katholieke Universiteit Leuven, (February 1996).

[8] Gaubert S., "Théorie des Systèmes Linéaires dans les Dioïdes", Thèse, Ecoles des Mines de Paris, Paris, (1992).

[9] Ljung L., "System Identification: Theory for the User", Prentice-Hall, Inc., Englewood Cliffs, New Jersey, (1987).

[10] Olsder G.J., "On an Analogy of Minimal Realizations in Conventionnal and Discrete Event Dynamic Systems", Séminaire CNRS/CNET/INRIA, Issy-les-moulineaux, (juin 1987).

[11] Prou J.M., Wagneur E., "Decomposition of Periodic Series", in proceedings MSCA'96, Chania, Crête, (juin 1996).

[12] Wang L., Xu X., "On Minimal Realization of SISO DEDS over Max-Algebra", ECC 93, pp. 535-540, Groningen, (1993). 